\title{
Demonstration of User Interfaces for Querying in 3D Architectural Content in PROBADO3D
}

\author{
René Bernd ${ }^{1}$, Ina Blümel ${ }^{2}$, Harald Krottmaier ${ }^{1}$, Raoul Wessel $^{3}$, \\ and Tobias Schreck ${ }^{4}$ \\ 1 Graz University of Technology \\ ${ }^{2}$ German National Library of Science and Technology TIB \\ ${ }^{3}$ University of Bonn \\ 4 Technische Universität Darmstadt
}

\begin{abstract}
The PROBADO project is a research effort to develop Digital Library support for non-textual documents. The main goal is to contribute to all parts of the Digital Library workflow from content acquisition over semi-automatic indexing to search and presentation. PROBADO3D is a part of the PROBADO framework designed to support 3D documents, with a focus on the Architectural domain. This demonstration will present a set of specialized user interfaces that were developed for content-based querying in this document domain.
\end{abstract}

\section{Interfaces for Querying in 3D Architectural Data}

PROBADO3D supports search in metadata space, as well as in content-based space in 3D architectural data comprising models of buildings, and interior and exterior elements. Content-based search relies on domain specific indexing services generating descriptors of the building models during an offline indexing stage. The descriptors include global shape properties as well as connectivity information which describes the layout of rooms within the buildings [1] (cf. Figure 1(a) for an example). For querying in these indexes, specific interfaces have been developed to graphically specify queries for similar content. The following interfaces are currently considered.

Querying for Similar Global Shape. Queries for the overall shape of building models are supported by sketching of $3 \mathrm{D}$ volumes, or by $2 \mathrm{D}$ floor plans. Two editors allow specification of volumes or floor plans. One interface provides a 2D/3D editor based on the Generative Modeling Language (GML [2]) (cf. Figure 1(b)). The other uses the modeling capabilities of Google Sketchup [3] (cf. Figure 1(c)).

Querying for Room Configurations. The configuration of rooms inside a building is also an important property which architects like to search for (cf. Figure 1(d)). A graph editor interface allows to input an abstract specification of room connectivity structure (cf. Figure 1(e)). A plan-based interface allows to edit a room sketch (cf. Figure 1(f)). 
The input provided by the user via these interfaces is then used to formulate content-based queries. A result list containing the models which best match the query are presented to the user for further inspection and browsing. The user can then refine the query by using any of the answer models as a query-by-example key.

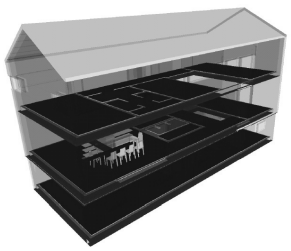

(a) Building model

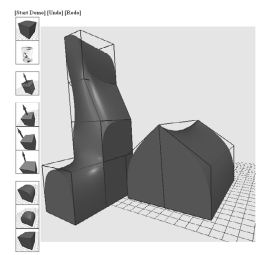

(b) Volume editor

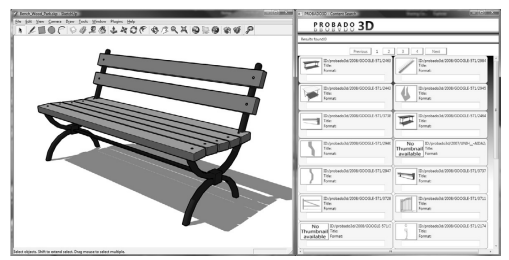

(c) Google Sketchup interface

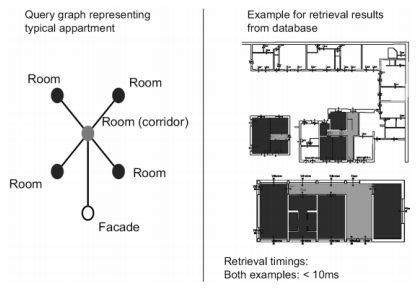

(d) Connectivity graph

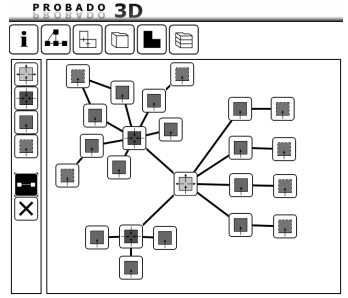

(e) Graph editor

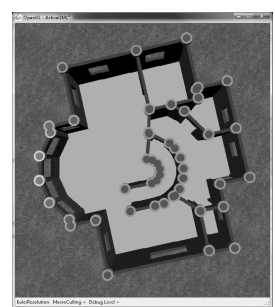

(f) Room editor

Fig. 1. Specialized graphical query interfaces support retrieval in the 3D Architectural model domain within PROBADO3D

\section{Acknowledgments}

We gratefully acknowledge support from the German Research Foundation DFG. The PROBADO project started in February 2006 with a tentative duration of five years. Partners are the University of Bonn, Technische Universitaet Darmstadt, the German National Library of Science and Technology in Hannover, and the Bavarian State Library in Munich. Cooperation partners are Graz University of Technology and Braunschweig Technical University. For further information, please refer to the PROBADO website at http://www.probado.de/. The work presented in this paper was partially supported under grants INST 9055/1-1, 1647/14-1, and 3299/1-1.

\section{References}

1. Wessel, R., Blümel, I., Klein, R.: The room connectivity graph: Shape retrieval in the architectural domain. In: Proc. Int. Conf. in Central Europe on Computer Graphics, Visualization and Computer Vision (2008)

2. GML: Generative modeling language (2009), http://www.generative-modeling . org/

3. Google: Google sketchup (2009), http://sketchup.google.com/ 